DARIA CORCOS $(*)$ - MASSIMILIANO CENTORAME $(*)$ - PIERFILIPPO CERRETTI $(*)$

\title{
DNA BARCODES REVEAL A NEW HOST RECORD FOR CARCELIA ATRICOSTA HERTING (DIPTERA TACHINIDAE) IN ITALY
}

\author{
(*) Department of Biology and Biotechnology "Charles Darwin”, Sapienza University of Rome, Piazzale Aldo Moro 5 , \\ 00185 , Rome, Italy. \\ Corresponding author: Daria Corcos (daria.corcos@uniroma1.it)
}

\begin{abstract}
Corcos D., Centorame M., Cerretti P. - DNA barcodes reveal a new host record for Carcelia atricosta Herting (Diptera Tachinidae) in Italy

The parasitoid-host association between Orgyia antiqua (Linnaeus) (Lepidoptera: Lymantriidae) and Carcelia atricosta Herting (Diptera: Tachinidae) is recorded here for the first time in Italy. A single caterpillar of O. antiqua was collected in Northern Italy (Arzergrande, Padua, Veneto Region) in June 2015. After the specimen died, a single tachinid larva emerged and pupariated. The emerged parasitoid was identified using DNA barcoding, with DNA extracted from the tachinid pupa. This is the first distributional record of C. atricosta in Northern Italy and the second for Italy, only two other specimens having being recorded previously (Abruzzo Region, Central Italy).
\end{abstract}

KeY WoRDS: Parasitoid, parasitoid-host association, host range, Lepidoptera, Orgyia antiqua, pupa.

\section{INTRODUCTION}

Tachinids are one of the largest and most diverse families of Diptera worldwide (STIREMAN et al., 2006). The adults usually feed on nectar and pollen, while the larvae are parasitoids of arthropods, mostly exophytic caterpillars and other herbivorous insects. Compared to hymenopteran parasitoids, tachinids are generally considered to have a broad host range (STIREMAN et al., 2006). However, recent studies show that their host range is extremely variable, with both polyphagous and specialized species (STIREMAN, 2016). Understanding the real extent of tachinid host ranges is extremely difficult because the family is taxonomically challenging, there are many undescribed and cryptic species, and rearing tachinids from all possible hosts poses practical problems (STIREMAN, 2005).

Orgyia antiqua (Linnaeus) (Lepidoptera: Lymantriidae), the rusty tussock moth, is a polyphagous moth native to Europe, but now introduced and widespread throughout Asia and North America. As with many other lymantriids, it can reach high population densities, becoming an important defoliator and pest of trees and cultivated plants. In Italy, $O$. antiqua is known to be parasitized by the tachinids Compsilura concinnata (Meigen) and Exorista larvarum (Linnaeus) (CERRETTI \& TSCHORSNIG, 2010); in the Palaearctic Region as a whole, it is parasitized by an additional 15 species of Tachinidae (TsCHORSNIG, 2017).

As part of a sampling study aimed at understanding the parasitoid community of phytophagous insects in a managed forest near Arzergrande (Padua, Veneto Region, Northern Italy), a single caterpillar of $O$. antiqua was collected on a blackberry shrub (Rubus sp.; Rosaceae) in June 2015. The specimen was reared and died after a few days. A tachinid larva emerged and pupariated immediately after the caterpillar died. After one year of rearing, an adult tachinid had not eclosed, and the puparium was thus placed in ethanol for preservation. DNA barcoding was used to identify the parasitoid.

\section{MATERIALS AND METHODS}

LABORATORY ANALYSIS

Genomic DNA was extracted and isolated by cutting out a section of the tachinid pupa using standard proteinase $\mathrm{K}-$ phenol/chloroform method with ethanol precipitation. The pupa has died and dried inside the puparium. Remains of the puparium and pupa have been deposited in the Museum of Zoology, Sapienza University of Rome, in Rome, Italy. The mitochondrial DNA fragment Cytochrome c Oxidase subunit I (COI) was amplified using two primer pairs: M13F-LCO (5'-TGTAAAACGACGGCCAGTGGTCAAC AAATCATAAAGATATTGG-3') and M13R-HCO (5' CAGGAAACAGCTATGACTAAACTTCAGGGTGACCA AAAAATCA-3') (FOLMER et al., 1994, modified). Amplification was carried out in $25 \mu 1$ reaction volume containing $50 \mathrm{mM}$ of $\mathrm{MgCl} 2,10 \mathrm{mM}$ of dNTP, $25 \mathrm{pM}$ of each primer, $0.75 \mathrm{U}$ Taq of Polymerase (Bioline), 1X NH4 reaction Buffer and 50ng of DNA. Cycling parameters were as follows: initial denaturation $\left(94^{\circ} \mathrm{C}, 5 \mathrm{~m}\right), 35$ cycles $\left(94^{\circ} \mathrm{C}\right.$, $\left.30 \mathrm{~s} ; 50^{\circ} \mathrm{C}, 30 \mathrm{~s} ; 72^{\circ} \mathrm{C}, 30 \mathrm{~s}\right)$ and final extension $\left(72^{\circ} \mathrm{C}, 10 \mathrm{~m}\right)$. PCR products were purified using Exosap-IT (USB Corporation) and sequenced by Macrogen Inc.

\section{Phylogenetic RECONSTRUCTION}

Consensus sequences were generated using Geneious R7.0.6 (Biomatters Inc.). Alignment was carried out using the ClustalW program in Geneious R7.0.6 with 26 sequences (Table 1). The Neighbor-joining clustering method was run with MEGA v.6 (KUMAR et al., 2008), using the default parameters.

\section{RESULTS}

The COI DNA sequence of the tachinid pupa was deposited in GenBank (NCBI) and is available under accession number MF539618. It was compared with all the sequences of species belonging to the Carcelia genus 
Table 1 - Sequences used to run the analysis which results are showed in Fig. I. Our sequence was compared with all COI sequences of the species belonging to the genus Carcelia RobineauDesvoidy available in GenBank (NCBI), excluding sequences without species identification. A minimum of three sequences for haplotype have been retained for each species.

\begin{tabular}{|c|c|c|}
\hline ID in tree & Species & Acc. Num. \\
\hline MF539618 & C. atricosta & MF539618 \\
\hline GU142048.1 & C. flavirostris & GU142048.1 \\
\hline GU142049.1 & C. flavirostris & GU142049.1 \\
\hline KM960164.1 & C. reclinata & KM960164.1 \\
\hline КР049202.1 & C. reclinata & KP049202.1 \\
\hline KP189254.1 & C. formosa & KP189254.1 \\
\hline KR390013.1 & C. atricosta & KR390013.1 \\
\hline KR428353.1 & C. reclinata & KR428353.1 \\
\hline KR435682.1 & C. reclinata & KR435682.1 \\
\hline KX843832.1 & C. puberula & KX843832.1 \\
\hline KX843855.1 & C. puberula & KX843855.1 \\
\hline KX843862.1 & C. lucorum & KX843862.1 \\
\hline KX843955.1 & C. lucorum & KX843955.1 \\
\hline KX844011.1 & C. gnava & KX844011.1 \\
\hline KX844177.1 & C. laxifrons & KX844177.1 \\
\hline KX844236.1 & C. atricosta & KX844236.1 \\
\hline KX844238.1 & C. tibialis & KX844238.1 \\
\hline KX844339.1 & C. lucorum & KX844339.1 \\
\hline KX844462.1 & C. atricosta & KX844462.1 \\
\hline KX844500.1 & C. tibialis & KX844500.1 \\
\hline KX844513.1 & C. tibialis & KX844513.1 \\
\hline KX844522.1 & C. bombylans & KX844522.1 \\
\hline KX844525.1 & C. bombylans & KX844525.1 \\
\hline KX844543.1 & C. laxifrons & KX844543.1 \\
\hline KX844474.1 & C. rasa & KX844474.1 \\
\hline HQ548469.1 & Blepharipa sp. & HQ548469.1 \\
\hline
\end{tabular}

available in GenBank [July 2017], revealing a similarity of $100.0 \%$ with Carcelia atricosta Herting (Diptera: Tachinidae) (Fig. I). Other than C. atricosta, eight additional species belonging to the same genus have been recorded in Italy: Carcelia alpestris Herting, Carcelia bombylans Robineau-Desvoidy, Carcelia dubia (Brauer \& Bergenstamm), Carcelia gnava (Meigen), Carcelia laxifrons Villeneuve, Carcelia lucorum (Meigen), Carcelia rasa (Macquart), and Carcelia rasella Baranov. Among them, $C$. gnava and $C$. rasa are known as parasitoids of $O$. antiqua in the Palaearctic Region (TSCHORSNIG, 2017). Both species were included in the analysis.
Carcelia atricosta is scattered distributed throughout Europe from the Mediterranean to Norway (PAPE et al., 2015). Only two specimens have been previously collected in Italy, both captured in Malaise traps in Central Italy (Collelongo site- Selva Piana (AQ), Abruzzo Region; 3-17 August 2004; lat. $41.8930^{\circ}$, long. $13.5968^{\circ}$; $1500 \mathrm{~m}$; legit. M. Romano; collection P. Cerretti, Museum of Zoology, Sapienza University, Rome, Italy) (CERRETTI, 2010). This represents the first record for Northern Italy (Arzergrande site (PD), Veneto Region; 26 June 2015; lat. 45.2565 , long. $12.0551^{\circ}, 8 \mathrm{~m}$; legit. D. Corcos).

\section{DISCUSSION}

The association between Orgyia antiqua and Carcelia atricosta had already been reported for the Czech Republic, the Netherlands and the United Kingdom (TSCHORSNIG, 2017 and literature therein), but is here recorded for the first time for Italy. Other known lepidopteran hosts of $C$. atricosta are: Orgyia recens Hübner (Lymantriidae), Malacosoma neustria Linnaeus (Lasiocampidae) and Acronicta psi Linnaeus (Noctuidae) (TsCHORSNIG, 2017).

The importance of tachinids as natural enemies of phytophagous pest insects is well documented. However, tachinid-host associations are still poorly understood (STIREMAN, 2016), in part because of the difficulties in reproducing the optimal conditions for rearing specimens in the laboratory. The identification of tachinid larvae or puparia based on morphological characters is seldom possible. As an alternative to morphological identifications of these lesser known life stages, the increasing number of COI sequences of tachinids in molecular libraries are creating a growing inventory of data that allows for the rapid and affordable identification of taxa (POHJOISMAKI et al., 2016). The use of molecular tools, as well as the availability of DNA sequences online, can dramatically improve our knowledge of parasitoid-host associations, especially in the case of rare or poorly-known species. Investigating the degree of tachinid host specificity and how widespread species conserve or change their host species in different regions, may help us to better understand the forces driving the diversification and evolution of these parasitoids (STIREMAN 2005). Also, because of their importance as enemies of pest insects, improving our knowledge of tachinid-host associations may be particularly useful in planning successful biological control programs (STIREMAN et al., 2006).

\section{REFERENCES}

Cerretti P., 2010 - I Tachinidi della fauna italiana (Diptera Tachinidae), con chiave interattiva dei generi ovest-paleartici. Volume I e II. Cierre Edizioni, Verona.

Cerretti P., Tschorsnig H.P., 2010 - Annotated host catalogue for the Tachinidae (Diptera) of Italy. Stuttgarter Beiträge zur Naturkunde A. Neue Serie, 3: 305-340.

Folmer O., Black M., Hoeh W., Lutz R., VRijenhoek R., 1994 - DNA primers for amplification of mitochondrial cytochrome c oxidase subunit I from diverse metazoan invertebrates. - Molecular Marine Biology and Biotechnology, 3: 294-299.

Kumar S., NeI M., Dudley J., Tamura K., 2008 - MEGA: a biologist-centric software for evolutionary analysis of DNA and protein sequences. - Briefings in Bioinformatics, 9: 299-306. 
Fig. I - Phylogenetic tree based on $543 \mathrm{bp}$ of the COI gene built with the Neighbor Joining method. Our sequence (MF539618) clusters with available sequences for C. atricosta sharing the same haplotype.

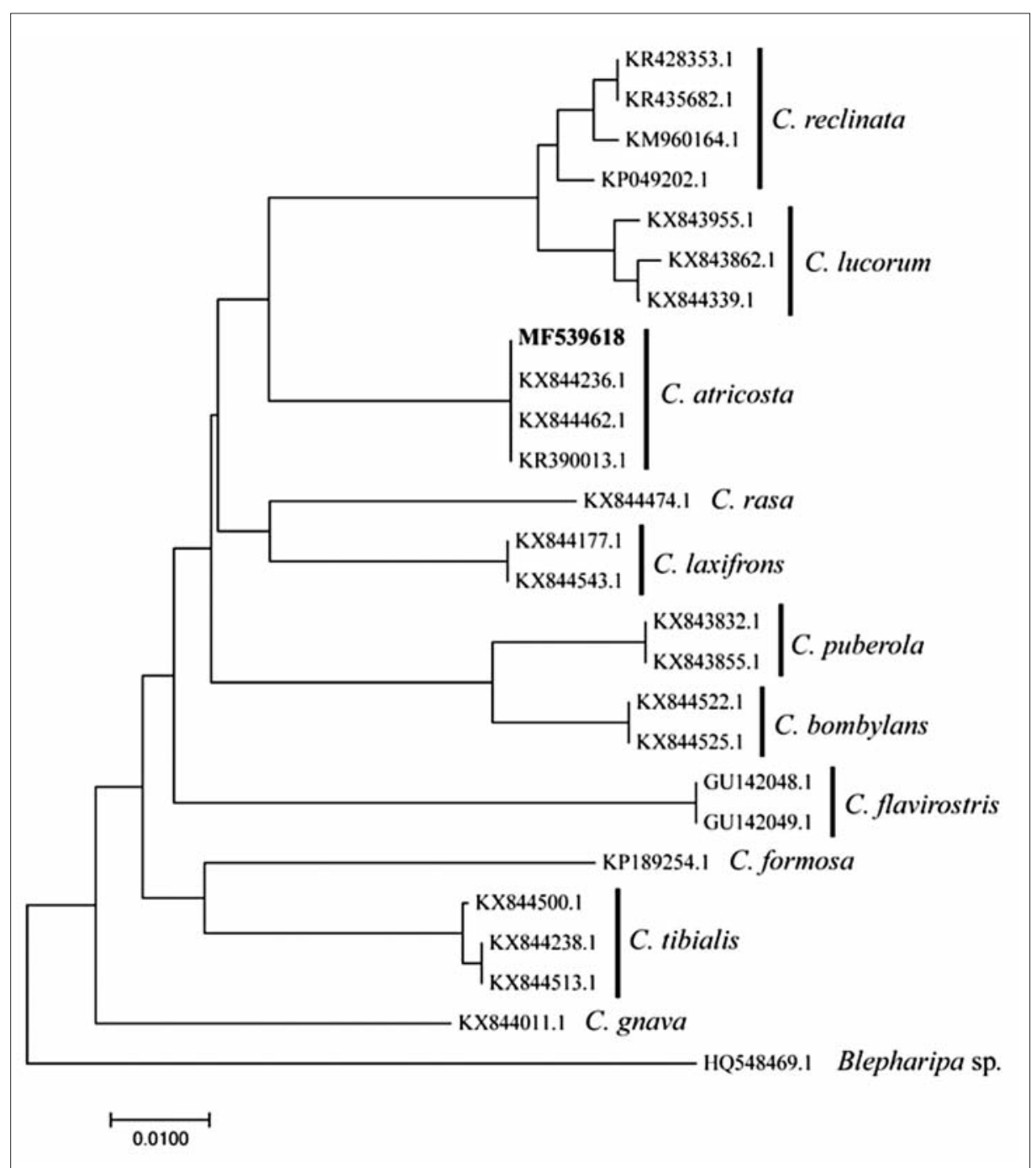

PAPE T., et al., 2015 - Fauna Europaea: Diptera Brachycera. - Biodiversity Data Journal, 3: e4187.

POHJOISMÄKI J.L.O., KAHANPÄÄ J., MutANEN M., 2016 DNA barcodes for the Northern European tachinid flies (Diptera: Tachinidae). - PLoS ONE, 11(11): e0164933.

StiREMAn J.O. III, 2005 - The evolution of generalization? Parasitoid flies and the perils of inferring host range evolution from phylogenies. - Journal of Evolutionary Biology, 18: 325-336.

STIREMAn J.O. III, 2016 - Community ecology of the 'other' parasitoids. - Current Opinion in Insect Science, 14: 8793.

Stireman J.O. III, O’hara J.E., WoOd D.M., 2006 Tachinidae: evolution, behavior, and ecology.- Annual Review of Entomology, 51: 525-555.

TSCHORSNIG H.-P., 2017 - Preliminary host catalogue of Palaearctic Tachinidae (Diptera). Available at: http://www.nadsdiptera.org/Tach/WorldTachs/CatPalHost s/Home.html. 
12 - Blank Page 